\title{
A LOWER BOUND FOR THE NUMBER OF ODD-DEGREE REPRESENTATIONS OF A FINITE GROUP
}

\author{
NGUYEN NGOC HUNG, THOMAS MICHAEL KELLER, AND YONG YANG
}

\begin{abstract}
Let $G$ be a finite group and $P$ a Sylow 2-subgroup of $G$. We obtain both asymptotic and explicit bounds for the number of odd-degree irreducible complex representations of $G$ in terms of the size of the abelianization of $P$. To do so, we, on one hand, make use of the recent proof of the McKay conjecture for the prime 2 by Malle and Späth, and, on the other hand, prove lower bounds for the class number of the semidirect product of an odd-order group acting on an abelian 2-group.
\end{abstract}

\section{INTRODUCTION}

This paper is concerned with the problem of bounding the number of $p^{\prime}$-degree irreducible (complex) representations of a finite group in terms of its $p$-local structure, where $p$ is a prime.

The number of irreducible representations of $p^{\prime}$-degree of a finite group is one of the important invariants that is predicted to be determined locally from the $p$-local information of the group. Indeed, the well-known McKay conjecture [Mc72, IMN07] asserts that

$$
\left|\operatorname{Irr}_{p^{\prime}}(G)\right|=\left|\operatorname{Irr}_{p^{\prime}}\left(\mathbf{N}_{G}(P)\right)\right|,
$$

where $\mathbf{N}_{G}(P)$ is the normalizer of a Sylow $p$-subgroup $P$ in $G$ and, as usual, $\operatorname{Irr}_{p^{\prime}}(G)$ denotes the set of irreducible representations of $p^{\prime}$-degree of $G$. Although the conjecture is still unsolved in general, it was confirmed for all finite groups and the prime $p=2$, thanks to the work Malle and Späth [MS16].

Note that every irreducible character of $\mathbf{N}_{G}(P)$ of degree coprime to $p$ must lie over a linear character of $P$. Moreover, every irreducible character of $\mathbf{N}_{G}(P) / P^{\prime}$ has $p^{\prime}$-degree. Therefore,

$$
\left|\operatorname{Irr}_{p^{\prime}}\left(\mathbf{N}_{G}(P)\right)\right|=\left|\operatorname{Irr}_{p^{\prime}}\left(\mathbf{N}_{G}(P) / P^{\prime}\right)\right|=\left|\operatorname{Irr}\left(\mathbf{N}_{G}(P) / P^{\prime}\right)\right|,
$$

which is the same as $k\left(\mathbf{N}_{G}(P) / P^{\prime}\right)$, the number of conjugacy classes of $\mathbf{N}_{G}(P) / P^{\prime}$.

2010 Mathematics Subject Classification. Primary 20C15, 20D10, 20 D05.

Key words and phrases. finite groups, odd-degree representations, characters, coprime action.

This work was initiated when the first author visited the Department of Mathematics at Texas State University during Fall 2017, and he would like to thank the department for its hospitality. The third author is partially supported by a grant from the Simons Foundation (No 499532). 
In view of [MS16], in this paper, we focus on the prime $p=2$ and attempt to bound $\left|\operatorname{Irr}_{2^{\prime}}(G)\right|$ in terms of $\left|P / P^{\prime}\right|$ - the size of the abelianization of $P$. Our first result is an asymptotic bound.

Theorem 1.1. Let $G$ be a finite group and $P$ a Sylow 2-subgroup of $G$. Then the number of odd-degree irreducible representations of $G$ is at least $\left|P / P^{\prime}\right|^{\alpha}$, where $\alpha>0$ is a universal constant.

The aforementioned result of Malle and Späth allows us to reduce Theorem 1.1 to the problem on bounding the class number of a semidirect product of an odd-order group acting on an abelian 2-group. We then make use of Feit-Thompson's theorem on solvability of odd-order groups, the Hartley-Turull lemma [HT94, Lemma 2.6.2] on isomorphic coprime group actions, and a result of the second-named author ([Ke11, Theorem B]) on bounding class numbers of solvable groups with trivial Frattini subgroup. Unfortunately, this approach does not provide us an explicit value of the bounding constant $\alpha$.

The problem of bounding the class number $k(G)$ of a finite group $G$ in terms of $|G|$ is important in Group Theory and has a long history, dating back to the work of Landau [L1903]. Though it has been studied extensively in the literature, it is still open whether $k(G)$ is logarithmically bounded below by $|G|$ (in fact, it has been believed that $k(G) \geq \log _{3}|G|$ for every $G$ ), see [Ke11, Ma16, BMT17] for the latest results and discussion.

Combining the class number conjecture with the McKay conjecture, we see that the number of $p^{\prime}$-degree irreducible characters of a finite group $G$ should be bounded below by $\log _{3}\left|P / P^{\prime}\right|$ (or even $\log _{2}\left|P / P^{\prime}\right|$ as in the case $p=2$ below), where $P$ is a Sylow $p$-subgroup of $G$. We confirm this expectation for $p=2$. The following result, although asymptotically weaker than Theorem 1.1, offers an explicit bound (and much better bound when $\left|P / P^{\prime}\right|$ is small) for $\left|\operatorname{Irr}_{2^{\prime}}(G)\right|$.

Theorem 1.2. Let $G$ be a finite group and $P$ a Sylow 2-subgroup of $G$. Then the number of odd-degree irreducible representations of $G$ is greater than $\log _{2}\left(\left|P / P^{\prime}\right|\right)$.

Theorem 1.2 can be considered as a generalization of a classical result of Burnside's theorem stating that every even-order group has a nontrivial irreducible representation of odd degree.

As explained above, Theorems 1.1 and 1.2 are consequences of the following result on bounding the class number.

Theorem 1.3. Let $G$ be an odd-order group acting on an abelian group $A$ of order $2^{n}$. Then $k(G A)>n$. Moreover, $k(G A) \geq 2^{\alpha n}$ where $\alpha>0$ is a universal constant.

Let $f(n)$ be the best bounding function in Theorem 1.3. To give the reader a sense of this function at some small values, we have found that $f(0)=1, f(1)=2$, $f(2)=4, f(3)=8, f(4)=8, f(5)=16, f(6)=16$, and $f(7)=32$. This suggests 
that the values of $f$ are perhaps always 2-powers, and it would be interesting to confirm this phenomenon.

Proving $p$-odd versions of Theorems 1.1, 1.2 and 1.3 appears to be a hard problem, one reason being that the group $G$ in Theorem 1.3 is not necessarily solvable anymore. However, although several results we prove along the way are specified to odd-order group actions only, we expect that the ideas can be developed further to treat other groups.

To end this introduction we remark that Malle and Maróti [MM16] have recently proved that $\left|\operatorname{Irr}_{p^{\prime}}(G)\right| \geq 2 \sqrt{p-1}$ for every prime $p$ and every finite group $G$ of order divisible by $p$. Our proposed bound $\left|\operatorname{Irr}_{p^{\prime}}(G)\right|>\log _{2}\left|P / P^{\prime}\right|$ is consistent with, but in many cases significantly stronger than, Malle-Maróti's bound, as shown by the case $p=2$ in Theorem 1.2.

\section{SOME PRELIMINARY RESULTS}

In this section we collect some preliminary lemmas that will be needed in the proof of the main results.

We start with a well-known result on bounding the order of an odd-order permutation group.

Lemma 2.1. Let $G$ be an odd-order subgroup of the symmetric group $\mathrm{S}_{m}$. Then $|G| \leq(\sqrt{3})^{m-1}$.

Proof. See [Di67] for a group-theoretic proof and [A167] for a combinatorial proof. Note that the equality holds if and only if $m$ is a power of 3 and $G$ is a Sylow 3-subgroup of $\mathrm{S}_{m}$.

The next two lemmas are important properties of odd-order group actions.

Lemma 2.2. Let $H$ be a (solvable) group of odd order, and let $V$ be a finite, faithful and irreducible $H$-module. Then

(i) $|V|$ cannot be the square of a Mersenne prime;

(ii) if $|V|=25$, then $|H|=3$;

(iii) if $|V|=81$, then $|H|=5$.

Proof. This follows from [Do05, Lemma 2.4].

Lemma 2.3. Let $G$ be an odd-order group and $V \neq 0$ be a faithful, completely reducible and finite $G$-module. If char $(V)$ is odd, then $|G| \leq|V|^{1.5} /(24)^{1 / 3}$.

Proof. This follows from [MW93, Theorem 3.5].

As usual we use $\mathbf{F}(G)$ to denote the Fitting subgroup of $G$, which is the minimal nilpotent normal subgroup of $G$, and $\operatorname{Soc}(G)$ to denote the subgroup generated by all minimal normal subgroups of $G$, which is usually referred to as the socle of $G$.

The following provides detailed structure of an odd-order group that acts faithfully, irreducibly, and quasi-primitively on a finite vector space. 
Lemma 2.4. Suppose that $V$ is a faithful, irreducible, and quasi-primitive module for an odd-order group $G$ over the Galois field $G F(q)$, where $q$ is a prime power. Then $G$ has normal subgroups $Z \subseteq T \subseteq \mathbf{F}(G) \subseteq A \subseteq G$ such that:

(i) $F:=\mathbf{F}(G)$ is a central product $F=E T$ with $E, T \triangleleft G$ and $T$ is cyclic, $Z=E \cap T=\mathbf{Z}(E)=\operatorname{Soc}(\mathbf{Z}(F))$ and $T=\mathbf{Z}(F)$, and Sylow subgroups of $E$ are of prime order or extra-special of prime exponent;

(ii) $A=\mathbf{C}_{G}(Z)$ and, in particular, $G / A$ is isomorphic to a subgroup of $\operatorname{Aut}(Z)$;

(iii) $T=\mathbf{C}_{G}(E), \mathbf{F}(G)=\mathbf{C}_{A}(E / Z)$, and $E / Z$ is a faithful completely reducible symplectic $A / \mathbf{F}(G)$-module;

(iv) $|F: T|=e^{2}$ where $e$ is an integer dividing $\operatorname{dim}(V)$;

(v) if $W$ is an irreducible $T$-submodule of $V$, then $T$ acts fixed point freely on $W$ and thus $|T|$ divides $|W|-1$;

(vi) $|V|=|W|^{\text {be }}$ for some integer $b$ and $|G: A| \mid \operatorname{dim}(W)$.

Proof. From the hypothesis and by the Clifford theorem, every normal abelian subgroup of $G$ is cyclic. The lemma then follows from [MW93, Corollary 1.10] and [MW93, Corollary 2.6].

Lemma 2.5. Suppose that $G$ is an odd-order (solvable) irreducible subgroup of $\operatorname{GL}(n, q)$ and the action of $G$ on the natural vector space is quasi-primitive. Let e be defined as in Lemma 2.4. Then $e \neq 3,7$.

Proof. Assume by contradiction that $e=3$ or $e=7$. We will keep the notation as in Lemma 2.4. We have $\mathbf{F}(G) / T \cong E / Z$ is a direct sum of the chief factors of $G$ and each factor, as an irreducible symplectic $G$-module, has even dimension (see [MW93, Corollary 1.10]). It follows that $G / \mathbf{C}_{G}(\mathbf{F}(G) / T)$ is a solvable irreducible subgroup of $\operatorname{GL}(2,3)$ or $\mathrm{GL}(2,7)$. Using Lemma 2.2 , we deduce that $\left|G / \mathbf{C}_{G}(\mathbf{F}(G) / T)\right|$ is even, a contradiction.

We need to use semi-linear groups and, for the reader's convenience, we recall the definition here.

Let $V$ be the Galois field $G F\left(q^{m}\right)$ for a prime power $q$. For fixed $a \in V^{\#}:=V \backslash\{0\}$, $w \in V$ and $\sigma \in \operatorname{Gal}\left(G F\left(q^{m}\right) / G F(q)\right)$, one can define the mapping

$$
T: V \rightarrow V \text { by } T(x)=a x^{\sigma}+w,
$$

which turns out to be a permutation on $V$. Note that $T$ is trivial if and only if $a=1$, $\sigma=1$ and $w=0$. The affine semi-linear group of $V$ is defined to be the group of all those permutations, and the semi-linear group is a subgroup of the affine semi-linear group:

$$
\Gamma(V)=\Gamma\left(q^{m}\right)=\left\{x \rightarrow a x^{\sigma} \mid a \in G F\left(q^{m}\right)^{\#}, \sigma \in \operatorname{Gal}\left(G F\left(q^{m}\right) / G F(q)\right)\right\} .
$$

To end this section, we record a result of S. Seager [Se88, Theorem 1]. We restate it here in the form suitable for our purposes. 
Theorem 2.6. Let $p$ be a prime and $G$ a finite solvable group acting faithfully and irreducibly on the finite $G F(p) G$-module $V$. Write $n(G, V)$ for the number of orbits of $G$ on $V$. Then by a classical result (see [Se88, Theorem 6]) we know that $G$ is isomorphic to a subgroup of $T$ ( $S$, where $T$ is a solvable irreducible subgroup of $\mathrm{GL}(m, p)$ and $S$ is a solvable transitive subgroup of $\mathrm{S}_{n}$ for some integers $m, n$; moreover, $\operatorname{dim} V=m n$. Then the following hold.

(i) If $T$ (as a linear group) is isomorphic to a subgroup of $\Gamma\left(p^{m}\right)$, then

$$
n \leq 0.157 \log _{3}\left((n(G, V)+1.43) / 24^{1 / 3}\right) .
$$

(ii) If $T$ (as a linear group) is not isomorphic to a subgroup of $\Gamma\left(p^{m}\right)$, then

$$
|V|=p^{m n}<\left((n(G, V)+1.43) / 24^{1 / 3}\right)^{c}
$$

$$
\text { for } c=36.435663 \text {. }
$$

Proof. This follows from [Se88, Section 6] by changing the language of primitive permutation groups to the language of linear groups.

\section{The ASYMptotic BOUnd}

In this section we prove Theorem 1.1. We start with the asymptotic part of Theorem 1.3. We thank the referee for pointing out this to us.

Theorem 3.1. Let $G$ be an odd-order group acting on an abelian 2-group $A$. Then $k(G A) \geq|A|^{\alpha}$ for some universal constant $\alpha>0$.

Proof. First we observe that $G$ is solvable by Feit-Thompson's theorem [FT63].

Suppose that the action of $G$ on $A$ is not faithful. Let $N:=\mathbf{C}_{G}(A)$. Then $1<N \unlhd G A$ and $G / N$ acts faithfully on $A$. We then have $k(G A) \geq k((G A) / N)=$ $k((G / N) A)$. So we may assume that $G$ is faithful on $A$. It follows that $A$ is the Fitting subgroup of $G A$.

A well-known formula for $k(G A)$ states that

$$
k(G A)=\sum_{i=1}^{n(G, A)} k\left(\mathbf{C}_{G}\left(a_{i}\right)\right),
$$

where $n(G, A)$ is the number of orbits of $G$ on $A$, and the $a_{i} \in A(1 \leq i \leq n(G, A))$ are representatives of the orbits of $G$ on $A$, see [Sc07, Proposition 3.1] for instance.

Now by the Hartley-Turull Lemma [HT94, Lemma 2.6.2], there exists an elementary abelian group $B$ such that the actions of $G$ on $A$ and $B$ are permutation isomorphic. Now $|A|=|B|$ and $n(G, A)=n(G, B)$, and if $\phi: A \rightarrow B$ is the corresponding permutation isomorphism, then $\mathbf{C}_{G}(a)=\mathbf{C}_{G}(\phi(a))$ for every $a \in A$. Hence by the $k(G A)$-formula above it follows that $k(G A)=k(G B)$, and so by replacing $A$ by $B$ if necessary we may assume that $A$ is elementary abelian. Therefore the Frattini subgroup of $G A$ is trivial. 
We have shown that $G A$ is a solvable group with trivial Frattini subgroup. Now using [Ke11], we deduce that there exists a universal constance $\alpha>0$ such that

$$
k(G A) \geq|G A|^{\alpha} \geq|A|^{\alpha},
$$

as desired.

Proof of Theorem 1.1. As explained in the introduction, we have

$$
\left|\operatorname{Irr}_{2^{\prime}}(G)\right|=k\left(\mathbf{N}_{G}(P) / P^{\prime}\right),
$$

by Malle-Späth's theorem. By the Schur-Zassenhaus theorem, $\mathbf{N}_{G}(P) / P^{\prime}$ is a split extension of the abelian 2-group $P / P^{\prime}$ and the odd-order group $\mathbf{N}_{G}(P) / P$. Therefore Theorem 1.1 follows by Theorem 3.1.

\section{TOWARD THE EXPLICIT BOUND}

We have to work much harder to obtain an (good) explicit bound for $\left|\operatorname{Irr}_{2^{\prime}}(G)\right|$. The main result of this section is Theorem 4.3, which will be used in a key step in the proof of Theorem 1.3 in the final section.

Recall that an orbit $x^{G}$ of an action of a group $G$ on a set $X$ is said to be regular if $\left|x^{G}\right|=|G|$, or equivalently, $\mathbf{C}_{G}(x)$ is trivial.

It was proved in [Ya12] that if $V$ is a finite, faithful, irreducible, and quasi-primitive $G$-module for an odd-order group $G$ with $\mathbf{F}(G)$ nonabelian (i.e. the action of $G$ on $V$ is not semi-linear), then $G$ has at least 212 regular orbits on $V$. (The bound 212 is indeed sharp.) In the following important result, we improve this to a bound in terms of $\operatorname{dim}(V)$.

Theorem 4.1. Let $G$ be a (solvable) group of odd order. Let $V$ be a finite, faithful, irreducible, and quasi-primitive $G$-module. Assume that the action of $G$ on $V$ is not semi-linear. Then $G$ has at least $\max (212, k+1)$ regular orbits on $V$, where $k=\operatorname{dim}(V)$.

Proof. By [Ya12, Theorem 1.1], we may assume that $\operatorname{dim}(V)=k \geq 212$. We will follow some ideas in the proof of [Do05, Theorem 2.2]. Assume that $G$ is a counterexample of minimal order. Then $G$ has at most $k$ regular orbits on $V$.

Let $G^{\#}$ be the set of nonidentity elements of $G$. Any $v \in V$ not contained in $\bigcup_{g \in G^{\#}} \mathbf{C}_{V}(g)$ must necessarily lie in a regular $G$-orbit, each of which has size exactly $|G|$. Since $G$ has at most $k$ regular orbits, we deduce that

$$
|V| \leq k \cdot|G|+\left|\bigcup_{g \in G^{\#}} \mathbf{C}_{V}(g)\right| .
$$

By [Es91, Lemma 1.3], we have

$$
\operatorname{dim}\left(\mathbf{C}_{V}(g)\right) \leq \frac{4}{9} \operatorname{dim}(V)
$$


whenever $g \neq 1$, and it follows that

$$
\left|\mathbf{C}_{V}(g)\right| \leq|V|^{\frac{4}{9}}
$$

for all $g \neq 1$.

Let $\mathcal{P}$ be the collection of all subgroups of $G$ of prime order. Then, as $|G|$ is odd, we have $|\mathcal{P}| \leq(|G|-1) / 2$. It follows that

$$
\left|\bigcup_{g \in G^{\#}} \mathbf{C}_{V}(g)\right|=\left|\bigcup_{S \in \mathcal{P}} \mathbf{C}_{V}(S)\right| \leq \frac{|G|-1}{2} \cdot|V|^{\frac{4}{9}}
$$

We then get

$$
|V| \leq k \cdot|G|+\frac{|G|-1}{2} \cdot|V|^{\frac{4}{9}}
$$

and thus

$$
|G| \geq \frac{2|V|+|V|^{\frac{4}{9}}}{2 k+|V|^{\frac{4}{9}}}>\frac{2|V|}{2 k+|V|^{\frac{4}{9}}} .
$$

We now adopt the notation in Lemma 2.4.

From Lemma 2.4 we know that $T$ acts fixed point freely on $W$ and $|T| \mid(|W|-1)$, thus $|T|<|W|$. Moreover $|T|$ is odd and $T \neq 1$, we also have $|T| \geq 3$ and therefore $|W| \geq 4$. Lemma 2.4(6) then implies that $|V| \geq 4^{e}$. Recall that $\operatorname{dim} V=k$, so $|V| \geq 2^{k}$. Therefore we have

$$
|V| \geq \max \left\{4^{e}, 2^{k}\right\}
$$

Note that $e$ is a positive odd integer bigger than 1 . In fact $e \geq 5$ by Lemma 2.5. This and the fact that $e \mid k$ imply that $\max \left\{4^{e}, 2^{k}\right\}^{4 / 9} \geq 2 k$. So we have

$$
|V|^{\frac{4}{9}}>2 k \text {. }
$$

Therefore

$$
|G|>|V|^{\frac{5}{9}}
$$

Since $|V|=|W|^{b e}$, we now have

$$
|G|>|W|^{\frac{5 b e}{9}} .
$$

Recall from Lemma 2.4(iii) that $F / T$ is a faithful completely reducible symplectic $A / F$-module. Using [Es91, Theorem A], we deduce that $F / T$ contains at least two regular $A / F$-orbits. Note that $|F / T|=e^{2}$. Hence

$$
|A / F| \leq|F / T| / 2=e^{2} / 2 .
$$

From Lemma 2.4(vi) that $|G / A| \mid \operatorname{dim} W$, we have $|G / A| \leq \log _{2}|W|$. Recall that $|T|<|W|$. We now have

$$
\begin{aligned}
|G| & =|G / A||A / F||F / T||T| \\
& <\log _{2}|W| \cdot e^{4} / 2 \cdot|W| .
\end{aligned}
$$


Therefore

$$
|W|^{5 e / 9}<\log _{2}|W| \cdot e^{4} \cdot|W| / 2 .
$$

Recall from Lemma 2.5 that $e \neq 3$ and $e \neq 7$. Let $p_{1}, \ldots, p_{l}$ be all the different prime divisors of $e$. Then $p_{1} \ldots p_{l}|| Z|||T| \mid(|W|-1)$. Take these into consideration and solve the above inequality, we come up with only two cases: $e=5,|W| \leq 70$ and $e=9,|W| \leq 10$.

(1) Assume $e=5$ and $|W| \leq 70$. Thus $|F / T|=e^{2}=25$ and $|A / F|=3$ by Lemma 2.2(ii). Moreover, $|G / A|$ is odd and $G / A \lesssim \operatorname{Aut}(Z)$. Since $|W|$ is a prime power and 5||$T||(|W|-1)$, we may assume $|T|=5$ or 15 .

(a) Assume $|T|=5$. Thus $Z \cong Z_{5}, G / A \lesssim \operatorname{Aut}(Z) \cong Z_{4}$ and hence $|G / A|=$ 1 as $|G / A|$ is odd. Therefore,

$$
|G|=|G / A||A / F||F / T||T|=3 \cdot 5^{3} .
$$

On the other hand, since $|T| \mid(|W|-1)$, we have $|W| \geq 11$. Also, since $\operatorname{dim}(V) \geq 5 \cdot \operatorname{dim}(W),|V| \geq 11^{5}$. But then we have $|G| \leq|V|^{5 / 9}$, a contradiction.

(b) Assume $|T|=15=5 \cdot 3$. Thus $Z \lesssim Z_{3} \times Z_{5}, G / A \lesssim \operatorname{Aut}(Z) \cong Z_{2} \times Z_{4}$ and again $|G / A|=1$. It follows that

$$
|G|=|G / A||A / F||F / T||T|=3 \cdot 5^{3} \cdot 3 .
$$

Since $|T| \mid(|W|-1)$, we have $|W| \geq 16$. Moreover, since $\operatorname{dim}(V) \geq$ $5 \cdot \operatorname{dim}(W)$, we get $|V| \geq 16^{5}$. But then we have $|G| \leq|V|^{5 / 9}$, a contradiction again.

(2) Assume $e=9$ and $|W| \leq 10$. Then $|F / T|=e^{2}=81$. Now the action of $A / F$ on $F / T$ is irreducible (since otherwise $|A / F|$ is even) and then $|A / F| \leq 5$ by Lemma 2.2. Since $|W|$ is a prime power and 3||$T||(|W|-1)$, we have $|W|=4$ or $7,|T|=3$ and $T=Z \cong Z_{3}$. It follows that $G / A \lesssim \operatorname{Aut}(Z) \cong Z_{2}$ and hence $G / A=1$.

Recall that $\operatorname{dim}(V) \geq 212$, we know that $|W|=7$, or $|W|=4$ and $b \geq 2$. We have $|G|=|G / A||A / F||F / T||T| \leq 5 \cdot 3^{5} \leq|V|^{5 / 9}$, a contradiction.

If $G$ is a permutation group on a set $\Omega$, there will be an induced action of $G$ on the power set $\mathscr{P}(\Omega)$. The $G$-orbit of a subset $\Delta \subseteq \Omega$ is regular if $\operatorname{Stab}_{G}(\Delta)$ is trivial. If we have $|\Delta| \neq|\Omega| / 2$ in addition, the orbit is called strongly regular.

Lemma 4.2. Let $G$ be an odd-order transitive primitive permutation group on $\Omega$ with $|\Omega|=n$. Then $G$ has at least $\left\lceil\frac{n}{25}\right\rceil$ strongly regular orbits on the power set $\mathscr{P}(\Omega)$ of $\Omega$.

Proof. We recall the structure of a solvable primitive permutation group. Let $V$ be a minimal normal subgroup of $G$ and let $S$ denote a point stabilizer. We know that 
$G=V S, S \cap V=1$, and $\mathbf{C}_{G}(V)=V$. Also $n=|\Omega|=|V|$ is a prime power, and we set $n=p^{t}$ where $p$ is a prime. For $g \in G$, we denote by $n(g)$ the number of cycles of $g$ on $\Omega$, by $o(g)$ the smallest prime divisor of the order of $g$, and by $s(g)$ the number of fixed points of $g$. We set $o$ to be the smallest order of all the nontrivial elements in $G$.

For a subset $X \subseteq G$, we consider the following set

$$
S(X)=\left\{(g, \Gamma) \mid g \in X, \Gamma \subseteq \Omega, g \in \operatorname{Stab}_{G}(\Gamma)\right\} .
$$

It is easy to see that in order to estimate $S(G \backslash\{1\})$, we only need to count elements of prime order. Also, assume $g_{1}$ is of prime order and let $g_{2} \in\left\langle g_{1}\right\rangle$, then the subsets of $\Omega$ stabilized by $g_{1}$ and $g_{2}$ are exactly the same. Note that $g \in G$ stabilizes exactly $2^{n(g)}$ subsets of $\Omega$.

Now we have

$$
|S(G \backslash\{1\})| \leq \frac{|G|}{o-1} \cdot 2^{\lfloor(p+o-1) n /(o \cdot p)\rfloor} .
$$

Therefore, it is sufficient to show that

$$
\frac{|G|}{o-1} \cdot 2^{\lfloor(p+o-1) n /(o \cdot p)\rfloor}<2^{n}-\left\lfloor\frac{n}{25}\right\rfloor \cdot|G|
$$

which is equivalent to

$$
|G|\left(\frac{\left.2^{\lfloor(p+o-1) n /(o \cdot p)}\right\rfloor}{o-1}+\left\lfloor\frac{n}{25}\right\rfloor\right)<2^{n} .
$$

Since $n$ is odd and $|G|$ is odd, we know that

$$
|G|=|V||S| \leq|V| \cdot|V|^{1.5} /(24)^{1 / 3}=n^{2.5} /(24)^{1 / 3}
$$

by Lemma 2.3. Moreover, $(p+o-1) /(o \cdot p) \leq 5 / 9$ as both $o$ and $p$ are at least 3 . Thus, it is sufficient to prove

$$
\frac{1}{24^{1 / 3}} \cdot n^{2.5}\left(\frac{2^{\left\lfloor\frac{5 n}{9}\right\rfloor}}{2}+\left\lfloor\frac{n}{25}\right\rfloor\right)<2^{n} .
$$

It is not hard to see that this inequality is satisfied when $n \geq 25$. When $n \leq 24$, we have $\left\lfloor\frac{n}{25}\right\rfloor=0$, and the result follows by Gluck's theorem that $G$ has at least one strongly regular orbit on $\mathscr{P}(\Omega)$, see [MW93, Theorem 5.6].

The following is the main result of this section, and it is a crucial step in the proof of Theorem 1.3.

Theorem 4.3. Let $G$ be a group of odd order. Let $V$ be a finite, faithful, and irreducible $G$-module. Assume $G$ is induced from a non-semi-linear group acting on $W \leq V$, then $G$ has at least $\max (n, 212)$ regular orbits on $V$, where $n=\operatorname{dim}(V)$. 
Proof. We first assume that $V$ is a quasi-primitive $G$-module. Thus $G$ is not semilinear. By Theorem 4.1, $G$ has at least $\max (n, 212)$ regular orbits on $V$.

Thus the action of $G$ on $V$ is not quasi-primitive and there exists a normal subgroup $N$ of $G$ such that $V_{N}=V_{1} \oplus \cdots \oplus V_{m}$ for $m>1$ homogeneous components $V_{i}$ of $V_{N}$. If $N$ is maximal with this property, then $S=G / N$ primitively permutes the $V_{i}$ 's. Also $V=V_{1}^{G}$, induced from $\mathbf{N}_{G}\left(V_{1}\right)$. Let $H=\mathbf{N}_{G}\left(V_{1}\right) / \mathbf{C}_{G}\left(V_{1}\right)$, then $H$ acts faithfully and irreducibly on $V_{1}$ and $G$ is isomorphic to a subgroup of $H$ ? $S$.

If $x_{1} \in V_{1}$ and $C_{1}$ is the $H$-conjugacy class of $x_{1}$, then the only $G$-conjugates of $x_{1}$ in $V_{1}$ are the elements of $C_{1}$. The set of $G$-conjugates of $x_{1}$ is $C_{1} \cup \cdots \cup C_{m}$ where $C_{i} \subset V_{i}$ is a $G$-conjugate of $C_{1}$. Choose $y_{1} \in V_{1}$ in an $H$-conjugate class different than that of $x_{1}$. Also choose $x_{i}$ and $y_{i}$ in $V_{i}$ conjugate to $x_{1}$ and $y_{1}$ (respectively) for all i. Then no $x_{i}$ is ever $G$-conjugate to a $y_{j}$. In particular, if $g \in G$ centralizes $v=x_{1}+\cdots+x_{j}+y_{j+1}+\cdots+y_{m}$, then $g$ and $N g$ must stabilize $\left\{V_{1}, \ldots, V_{j}\right\}$ and $\left\{V_{j+1}, \ldots, V_{m}\right\}$.

Set $\operatorname{dim}\left(V_{1}\right)=l$, and we know that $n=m \cdot l$. By induction, $H$ has at least $a=\max (l, 212)$ regular orbits on $V_{1}$. Say $u_{1}, v_{1}, w_{1}$ are three elements in distinct such orbits. Let $u_{i} \in V_{i}$ be $G$-conjugates of $u_{1}(1 \leq i \leq m)$, let $v_{i} \in V_{i}$ be $G$ conjugates of $v_{1}(1 \leq i \leq m)$, and let $w_{i} \in V_{i}$ be $G$-conjugates of $w_{1}(1 \leq i \leq m)$. Suppose that $\Omega$ can be written as a disjoint union of $A_{1}, A_{2}\left(A_{i} \neq \varnothing, i=1,2\right)$ such that $\operatorname{Stab}_{S}\left(A_{1}\right) \cap \operatorname{Stab}_{S}\left(A_{2}\right)=1$ and $\left|A_{1}\right|<\left|A_{2}\right|$. Define $x=x_{1}+x_{2}+\cdots+x_{m}$ such that $x_{i}=u_{i}$ if $i \in A_{1}$, and $x_{i}=v_{i}$ if $i \in A_{2}$. Then $\mathbf{C}_{G}(x)=\mathbf{C}_{N}(x)=1$. Define $y=y_{1}+y_{2}+\cdots+y_{m}$ such that $y_{i}=v_{i}$ if $i \in A_{1}$, and $y_{i}=u_{i}$ if $i \in A_{2}$. We see that $x$ is not $G$-conjugate to $y$ since $\left|A_{1}\right| \neq\left|A_{2}\right|$. Define $z=z_{1}+z_{2}+\cdots+z_{m}$ such that $z_{i}=u_{i}$ if $i \in A_{1}$, and $z_{i}=w_{i}$ if $i \in A_{2}$. Then $\mathbf{C}_{G}(z)=\mathbf{C}_{N}(z)=1$. It is easy to see that $x$ and $z$ are not $G$-conjugate. Thus using the pair $A_{1}$ and $A_{2}$ we may construct $a(a-1)$ regular orbits of $G$ on $V$.

We now suppose that $\Omega$ can be written as a disjoint union of $B_{1}, B_{2}\left(B_{i} \neq \varnothing\right.$, $i=1,2)$ such that $\operatorname{Stab}_{S}\left(B_{1}\right) \cap \operatorname{Stab}_{S}\left(B_{2}\right)=1$ and $\left|B_{1}\right|<\left|B_{2}\right|$. Then, in a similar way, we may construct $a(a-1)$ regular orbits of $G$ on $V$. We observe that as long as $A_{1}$ and $B_{1}$ are in different set-orbits of $G / N$ on the power set of $\Omega$, the regular orbits constructed using $A_{1}, A_{2}$ and the ones constructed using $B_{1}, B_{2}$ are in different $G$-orbits. By Lemma 4.2 , there are at least $\left\lceil\frac{m}{25}\right\rceil / 2$ such pairs. Thus $G$ has at least

$$
\frac{a(a-1) \cdot\left\lceil\frac{m}{25}\right\rceil}{2}
$$

regular orbits on $V$. Clearly

$$
\frac{a(a-1) \cdot\left\lceil\frac{m}{25}\right\rceil}{2} \geq \max (n, 212)
$$

as desired. 


\section{The EXPLICIT BOUND}

We are now ready to prove the explicit bound in Theorem 1.3, which is restated below.

Theorem 5.1. Let $G$ be an odd-order group acting on an abelian group $V$ of order $2^{n}$. Then $k(G V)>n$.

Proof. Let $(G, V$ be a counterexample such that $G V$ has minimal order. In particular, we have $k(G V) \leq n$. We proceed in a number of steps.

Step 1. $G$ acts faithfully on $V$.

As in the proof of Theorem 3.1, if $G$ is not faithful on $V$ then $1<\mathbf{C}_{G}(V) \unlhd G V$ and $G / \mathbf{C}_{G}(V)$ acts (faithfully) on $V$; moreover, by the choice of $G$ and $V$, we have $k((G V) / N)=k((G / N) V>n$, and since clearly $k(G V) \geq k((G V) / N)$, we obtain $k(G V)>n$, contradicting $G$ and $V$ being a counterexample. So $G$ is indeed faithful on $V$.

Step 2. $V$ is elementary abelian.

Using the $k(G V)$-formula $k(G V)=\sum_{i=1}^{n(G, V)} k\left(\mathbf{C}_{G}\left(v_{i}\right)\right)$ (where $n(G, V)$ is the number of orbits of $G$ on $V$ and the $v_{i} \in V(1 \leq i \leq n(G, V))$ are representatives of the orbits of $G$ on $V$ ) and the Hartley-Turull Lemma [HT94, Lemma 2.6.2], and arguing similarly as in the proof of Theorem 3.1, we may assume that $V$ is elementary abelian.

Step 3. $V$ is an irreducible $G$-module.

By Steps 1 and 2 we know that $V$ can be viewed as a faithful $G$-module over the field with two elements, and since $G$ acts coprimely on $V$, by Maschke's theorem we know that $V$ is completely reducible as $G$-module.

Now suppose that $V=X \oplus Y$ for two nontrivial $G$-modules $X$ and $Y$. Write $|X|=2^{s}$ and $|Y|=2^{t}$. Then $s+t=n, s \geq 1$ and $t \geq 1$. By interchanging $X$ and $Y$ if necessary we may assume that $s \leq t$. Also note that since $X$ is nontrivial, we have $n(G, X) \geq 2$. We now apply [Ke06, Lemma 4.3] to deduce that

$$
\begin{aligned}
k(G V) & =\sum_{i=1}^{n(G, X)} k\left(C_{G}\left(x_{i}\right) Y\right)>\sum_{i=1}^{n(G, X)} t \\
& =n(G, X) t \geq 2 t \geq s+t=n,
\end{aligned}
$$

where the $x_{i} \in X(1 \leq i \leq n(G, X))$ are representatives of the orbits of $G$ on $X$. This contradicts $(G, V)$ being a counterexample, and so we now have that $V$ is irreducible 
as a $G$-module.

Step 4. $V$ is induced from a semi-linear group action. That is, there exists a $H \leq G$ and an irreducible $H$-module $W$ such that $W<V, H / \mathbf{C}_{H}(W) \leq \Gamma(W)$, and $V=W^{G}$.

Assume not and that $H$ is not semi-linear, then the result follows by Theorem 4.3.

Step 5. $n \leq 15$.

Write $|W|=2^{k}$, so that $H / \mathbf{C}_{H}(W) \leq \Gamma\left(2^{k}\right)$ and hence $\left|H / \mathbf{C}_{H}(W)\right| \leq\left(2^{k}-1\right) k$. Also let $N:=\cap_{g \in G} H^{g} \unlhd G$ be the core of $H$ in $G$, then

$$
V_{N}=W_{1} \oplus \cdots \oplus W_{m}
$$

where $m=n / k$ and the $W_{i}$ s are homogeneous $N$-modules which are faithfully permuted by $G / N$, and we may assume that $W=W_{1}$. Hence $G / N$ is isomorphic to an odd order subgroup of $S_{m}$, and thus by Lemma 2.1 we have

$$
|G / N| \leq(\sqrt{3})^{m-1}
$$

Moreover, clearly $|N| \leq\left(2^{k}-1\right)^{m} k^{m} \leq 2^{n} k^{m}$, and $N$ has an abelian subgroup $K$ which is normal in $G$ and whose index in $N$ is at most $k^{m}$. Therefore $K V$ is a metabelian normal subgroup of $G$, and by [Be06, Theorem 1] we know that

$$
k(K V)>|K V|^{\frac{1}{3}} .
$$

We now get

$$
\begin{aligned}
n & \geq k(G V) \geq \frac{k(K V)}{|G V: K V|} \geq \frac{|K V|^{\frac{1}{3}}}{|G: K|} \\
& \geq \frac{|V|^{\frac{1}{3}}}{(\sqrt{3})^{m-1} k^{m}} \geq \frac{2^{\frac{n}{3}}}{(\sqrt{3} k)^{\frac{n}{k}}}>\frac{2^{\frac{n}{3}}}{(\sqrt{3} n)^{\frac{n}{k}}} .
\end{aligned}
$$

We next use Seager's result Theorem 2.6. Since $V$ is induced from a semi-linear group, we are in Part (a) of that result. Thus we have

$$
\begin{aligned}
\frac{n}{k} & \leq 0.157 \log _{3}\left((n(G, V)+1.43) / 24^{1 / 3}\right) \\
& <0.157 \log _{3}\left((k(G V)+1.43) / 24^{1 / 3}\right) \\
& \leq 0.157 \log _{3}\left((n+1.43) / 24^{1 / 3}\right) .
\end{aligned}
$$


Two inequalities in the last two paragraphs yields

$$
n>\frac{2^{\frac{n}{3}}}{(\sqrt{3} n)^{0.157 \log _{3}\left((n+1.43) / 24^{1 / 3}\right)},}
$$

and hence

$$
2^{\frac{n}{3}}<n \cdot(\sqrt{3} n)^{0.157 \log _{3}\left((n+1.43) / 24^{1 / 3}\right)} .
$$

This turns out to be true only when $n \leq 15$, as claimed.

Step 6. $n>k$, where we recall that $k=\log _{2}|W|$.

Suppose that $n=k$, so by Steps 4 and 5 we have $G \leq \Gamma\left(2^{n}\right)$ where $n \leq 15$. If $n=1$, we get a contradiction immediately, so we may assume that $n \geq 3$ (as $|G|$ is odd).

Write $\Gamma_{0}\left(2^{n}\right)$ for the cyclic subgroup of $\Gamma\left(2^{n}\right)$ consisting of scalar multiplications when considered acting on the field of $2^{n}$ elements. Let $K=G \cap \Gamma_{0}\left(2^{n}\right)$, so $K$ is cyclic and normal in $G$ of order dividing $2^{n}-1$ and acts frobeniusly on $V$. Let $l=|G / K|$, so $l$ divides $n$. If $l=n$, then we get $k(G V)>k(G V /(K V))=k(G / K)=l=n$, a contradiction. Therefore, since $|G|$ is odd and $n \geq 3$, we get $l \leq n / 3$.

Now we have

$$
\frac{n^{2}}{3} \geq l \cdot n \geq l k(G V) \geq k(K V) \geq|K|+\frac{|V|-1}{|K|} .
$$

It is an easy exercise to check that the function $f(x)=x+\frac{|V|-1}{x}(0<x<|V|)$ attains its minimum value at $x=\sqrt{|V|-1}$, and this minimum value is $2 \sqrt{|V|-1}$. It follows that

$$
\frac{n^{2}}{3} \geq 2 \sqrt{|V|-1}=\sqrt{4|V|-4} \geq \sqrt{3|V|}=\sqrt{3} \cdot 2^{n / 2} .
$$

(The last inequality holds since $n \geq 3$.) Thus $n^{2} \geq 3 \sqrt{3} \cdot 2^{n / 2}$, and it is easy to verify that this inequality holds for no positive integer $n$. This contradiction completes the proof of Step 6.

Step 7. Final contradiction.

We keep using the above notation. Clearly, the action of $G$ on $V$ will have at least $m=n / k$ distinct orbits, and each of these orbits is a conjugacy class of $G V$. Hence

$$
(++) \quad k(G V) \geq \frac{n}{k}+1,
$$

and we will use this as we go through the remaining small cases. Recall that $k(G V) \leq$ $n$, we must have $k>1$. 
By Steps 5 and 6 and the facts that $|G|$ is odd and $k>1$, the remaining cases that we have to look at are the following:

- $n=15, k=3$;

- $n=15, k=5$;

- $n=14, k=2$;

- $n=12, k=4$;

- $n=12, k=2$;

- $n=10, k=2$;

- $n=9, k=3$;

- $n=6, k=2$.

We now consider each of these cases separately.

$n=15, k=3$ : Since the only odd order subgroups of $S_{5}$ containing a 5 -cycle are the Sylow 5-subgroups, we have $G \lesssim \Gamma\left(2^{3}\right)>Z_{5}$ and $|G|$ divides $7^{5} \cdot 3 \cdot 5 \cdot 5$. We get six conjugacy classes from $(++)$ and four more from elements of order 5 . If $3^{3}$ divides $|G|$, then, as $Z_{5}$ permutes the elements of order three in orbits of lengths 1 and five, we get six more conjugacy classes of $G$ from elements of order 3, contradicting $k(G V) \leq n=15$. So $|G|$ is not divisible by $3^{3}$. In particular, the Hall $\{3,5\}$-subgroup of $G$ has order no more than 45 . If $7^{3}$ divides $|G|$, then we see that we get more than $7^{3} / 45$, that is, at least eight more classes of $G V$ from elements of order 7 , again leading to a contradiction to $k(G V) \leq 15$. Hence $|G|$ divides $5 \cdot 3^{2} \cdot 7^{2}$. Now $G$ acts on the nonzero elements of $V$, and hence $k(G V) \geq(|V|-1) /|G|+1 \geq 8^{5} /(45 \cdot 49)+1>15.5$, so $k(G V) \geq 16$, again a contradiction, concluding this case.

$n=15, k=5$ : Then $G \lesssim \Gamma\left(2^{5}\right) \succ Z_{3}$. From $(++)$ we get four classes of $G V$, and from elements of order three at least two more classes. If $5^{3}$ divides $G$, we get $\left(5^{3}-1\right) / 3>40$ more classes from elements of order 5 , a contradiction. So $|G|$ is not divisible by $5^{3}$. Also, if $31^{2}$ divides $|G|$, then we get at least $\left(31^{2}-1\right) /\left(5^{2} \cdot 3\right)>12$ conjugacy classes from elements of order 31, bringing the total for $k(G V)$ above 15 , a contradiction. Thus we now know that $|G|$ divides $3 \cdot 5^{2} \cdot 31$, and as in the previous case we then see that $k(G V) \geq(|V|-1) /|G|+1 \geq 8^{5} /(75 \cdot 31)>15$, the final contradiction in this case.

$n=14, k=2$ : Then by [MW93, Corollary 2.25] we know that $G \lesssim \Gamma\left(2^{2}\right) \curlywedge\left(Z_{7} \cdot Z_{3}\right)$, so by the oddness of $|G|$, we even have $G \lesssim Z_{3} 2\left(Z_{7} \cdot Z_{3}\right)$. From $(++)$ we get eight classes of $G V$ inside $V$. Bringing back the notation from Step 5, we recall that $V_{N}=W_{1} \oplus \cdots \oplus W_{7}$ for subspaces $W_{i}$ s which are transitively permuted by $G$, and we write $K$ for the kernel of this permutation action. Then $K$ is an elementary abelian 3 -group of order at most $3^{7}$, and $|G / K|$ divides 21 . Since clearly 7 divides $|G|$, from $G / K$, which is cyclic of order 7 or Frobenius of order 21, we get five conjugacy classes. 
Now if $3^{4}$ divides $|K|$, then we get an additional at least $\left(3^{4}-1\right) / 21$, that is three conjugacy classes of $G V$ coming from (nontrivial) elements in $K$, bringing the total above 14 , a contradiction. Hence $|K|$ divides $3^{3}$ and hence $|G|$ divides $27 \cdot 21$. But then as before we see that $k(G V) \geq(|V|-1) /|G| \geq\left(2^{14}-1\right) /(27 \cdot 21)>28$, which gives a final contradiction in this case.

$n=12, k=4$ : Then $G \lesssim \Gamma\left(2^{4}\right)\left\{Z_{3}\right.$ and then, as $|G|$ is odd, even $G \lesssim Z_{15}$ $Z_{3}$. From $(++)$ we get four classes, and from the " $Z_{3}$ on top" we get two additional classes. If $5^{2}$ divides $|G|$, then from elements of order 5 we get $\left(5^{2}-1\right) / 3=8$ more classes, bringing the number of conjugacy classes of $G V$ to 12 . So there cannot be any other elements in the group, forcing $|G|=5^{2} \cdot 3=75$. But then $k(G V) \geq(|V|-1) /|G|=$ $\left(2^{12}-1\right) / 75=4095 / 75>12$, a contradiction.

Now we have $5^{2}$ does not divide $|G|$. For the number $n(G, V)$ of orbits of $G$ on $V$ we have $n(G, V) \geq(|V|-1) /|G| \geq\left\lceil 4095 /\left(5 \cdot 3^{3} \cdot 3\right)\right\rceil=11$, which together with the two classes mentioned above from the factor group of order 3 increases the number of classes of $G V$ to more than 13, a contradiction.

$n=12, k=2$ : In this case $G / N$ would be a transitive permutation group of degree 6 and as such not be primitive, it follows that $W_{i}$ 's $(i=1, \ldots, 6)$ would split into three blocks of imprimitivity of size 2 or two blocks of size 3 . Since $G / N$ is of odd order, it could not act transitively on the $W_{i}$, a contradiction.

$n=10, k=2$ : Then $G \lesssim \Gamma\left(2^{2}\right) \imath Z_{5}$ and hence even $G \lesssim Z_{3} \imath Z_{5}$. By $(++)$ we have six classes of $G V$ in $V$, and from the factor group of order 5 we get an additional four classes, so these must be all conjugacy classes of $G V$, implying $|G|=5$, but then $k(G V) \geq(|V|-1) /|G| \geq 1023 / 5 \geq 11$, a contradiction.

$n=9, k=3$ : Here $G \lesssim \Gamma\left(2^{3}\right) \imath Z_{3}$, so by $(++)$ we get four classes of $k(G V)$. Again recall that $V_{N}=W_{1} \oplus W_{2} \oplus W_{3}$ for subspaces $W_{i}$ s which are transitively permuted by $G$, and $K$ is the kernel of this permutation action. Then $K$ is an abelian $\{3,7\}$-group of order at most $15^{3}$, and $G / K$ is cyclic of order 3. So from $G / K$ we get another two classes. Now if $3^{2}$ divides $|K|$, then from elements of order 3 in $K$ we get additional more than $8 / 3$, that is, three classes, implying $k(G V)=10>n$, a contradiction.

Therefore $3^{2}$ does not divide $|K|$. Now if $7^{2}$ divides $|G|$, then observe that we get at least 48/9, thus six classes from elements of order 7 , and together with the four classes from $(++)$ this again leads to a contradiction. Thus $7^{2}$ does not divide $|G|$, so $|G|$ divides 21 and thus $k(G V) \geq(|V|-1) /|G| \geq 511 / 21>10$, the final contradiction in this case.

$n=6, k=2$ : In this last case, $G \lesssim \Gamma\left(2^{2}\right)$ 乙 $Z_{3}$ and thus $G \lesssim Z_{3}$ 乙 $Z_{3}$. From $(++)$ we get four classes, and from the factor group of order three we get two more. This 
forces $k(G V)=6$ and moreover $|G|=3$. Now $k(G V) \geq(|V|-1) /|G| \geq 61 / 3>7$. This final contradiction concludes the proof of the theorem.

Proof of Theorem 1.2. Theorem 1.2 follows from Theorem 5.1 and [MS16, Theorem 1], as discussed in the Introduction.

\section{REFERENCES}

[Al67] B. Alspach, A combinatorial proof of a conjecture of Goldberg and Moon, Canad. Math. Bull. 11 (1968), 655-661.

[BMT17] B. Baumeister, A. Maróti, and H.P. Tong-Viet, Finite groups have more conjugacy classes, Forum Math. 29 (2017), 259-275.

[Be06] E. A. Bertram, Lower bounds for the number of conjugacy classes in finite groups, Ischia group theory 2004, 95-117, Contemp. Math., 402, Israel Math. Conf. Proc., Amer. Math. Soc., Providence, RI, 2006.

[Di67] J.D. Dixon, The maximum order of the group of a tournament, Canad. Math. Bull. 10 (1967), 503-505.

[Do05] S. Dolfi, Intersections of odd order Hall subgroups, Bull. Lond. Math. Soc. 37 (2005), $61-66$

[Es91] A. Espuelas, Regular orbits on symplectic modules, J. Algebra 138 (1991), 1-12.

[FT63] W. Feit and J. G. Thompson, Solvability of groups of odd order, Pacific J. Math. 13 (1963), 775-1029.

[HT94] B. Hartley and A. Turull, On characters of coprime operator groups and the Glauberman character correspondence, J. Reine Angew. Math. 451 (1994), 175-219.

[IMN07] I. M. Isaacs, G. Malle, and G. Navarro, A reduction theorem for the McKay conjecture, Invent. Math. 170 (2007), 33-101.

[Ke06] T.M. Keller, Fixed conjugacy classes of normal subgroups and the $k(G V)$-problem, $J$. Algebra 305 (2006), 457-486.

[Ke11] T. M. Keller, Finite groups have even more conjugacy classes, Israel J. Math. 181 (2011), 433-444.

[L1903] E. Landau, Über die Klassenzahl der binären quadratischen Formen von negativer Discriminante, Math. Ann. 56 (1903), 671-676.

[MS16] G. Malle and B. Späth, Characters of odd degree, Ann. of Math. (2) 184 (2016), 869-908.

[MM16] G. Malle and A. Maróti, On the number of $p^{\prime}$-degree characters in a finite group, Int. Math. Res. Not. 20 (2016), 6118-6132.

[MW93] O. Manz and T.R. Wolf, Representations of Solvable Groups, Cambridge University Press, 1993.

[Ma16] A. Maróti, A lower bound for the number of conjugacy classes of a finite group, Advances in Math. 290 (2016), 1062-1078.

[Mc72] J. McKay, Irreducible representations of odd degree, J. Algebra 20 (1972), 416-418.

[Se88] S. M. Seager, A bound on the rank of primitive solvable permutation groups, J. Algebra 116 (1988), 342-352.

[Sc07] P. Schmid, The solution of the $k(G V)$ problem, ICP Advanced Texts in Mathematics, 4. Imperial College Press, London, 2007.

[Ya12] Y. Yang, Orbits of the actions of odd-order solvable groups, Comm. Algebra 40 (2012), 565-574. 
Department of Mathematics, The University of Akron, Akron, OH 44325, USA

E-mail address: hungnguyen@uakron.edu

Department of Mathematics, Texas State University, 601 University Drive, San MARCOS, TX 78666, USA

E-mail address: keller@txstate.edu

Department of Mathematics, Texas State University, 601 University Drive, San MARCOS, TX 78666, USA

E-mail address: yang@txstate.edu 